\title{
Quadratic conservation laws and collineations: a discussion
}

\author{
Leonidas Karpathopoulos \\ Faculty of Physics, Department of Astronomy-Astrophysics-Mechanics, \\ University of Athens, Panepistemiopolis, Athens 157 83, Greece \\ Michael Tsamparlis* \\ Faculty of Physics, Department of Astronomy-Astrophysics-Mechanics, \\ University of Athens, Panepistemiopolis, Athens 157 83, Greece \\ Andronikos Paliathanasis ${ }^{\dagger}$ \\ Instituto de Ciencias Fúsicas y Matemáticas, \\ Universidad Austral de Chile, Valdivia, Chile \\ Institute of Systems Science, Durban University of Technology \\ Durban 4000, Republic of South Africa
}

\begin{abstract}
Every second order system of autonomous differential equations can be described by an autonomous holonomic dynamical system with a Lagrangian part, an effective potential and a set of generalized forces. The kinematic part of the Lagrangian defines the kinetic metric which subsequently defines a Riemannian geometry in the configuration space. We consider the generic function $I=K_{a b}\left(t, q^{c}\right) \dot{q}^{a} \dot{q}^{b}+K_{a}\left(t, q^{c}\right) \dot{q}^{a}+$ $K\left(t, q^{c}\right)$ and require the quadratic first integral condition $d I / d t=0$ without involving any type of symmetry Lie or Noether. Condition $d I / d t=0$ leads to a system of equations involving the coefficients $K_{a b}\left(t, q^{c}\right), K_{a}\left(t, q^{c}\right), K\left(t, q^{c}\right)$ whose solution will produce all possible quadratic first integrals of the original system of autonomous differential equations. We show that the new system of equations relates the quadratic first integrals of the holonomic system with the geometric collineations of the kinetic metric and in particular with the Killing tensors of order two. We consider briefly various results concerning the Killing tensors of second-order and prove a general formula which gives in the case of a flat kinetic metric the generic Killing tensor in terms of the vectors of the special projective algebra of the kinetic metric. This establishes the connection between the geometry defined by the kinetic metric and the quadratic first integrals of the original system of differential equations.
\end{abstract}

Keywords: Dynamical systems, Lie Symmetries, First integrals, Generalized symmetries PACS - numbers: 2.40.Hw, 4.20.-q, 4.20.Jb, 04.20.Me, 03.20.+i, 02.40.Ky

\section{Introduction}

At the end of the 19th century Sophus Lie published his work on the theory of transformation groups [1, 2, 3]. In particular he introduced the idea of transformations which leave invariant functions and geometric objects. The application of Lie's method has led to new interesting results is the area of differential equations.

A Lie symmetry of a differential equation generated by the vector $X=\xi(t, q, \dot{q}) \partial_{t}+\eta^{a}(t, q, \dot{q}) \partial_{q^{a}}$ is a point transformation in the jet space $J^{1}\left\{t, q^{a}, \dot{q}\right\}$ which preserves the form of the equation and transforms solutions into solutions. In this work we are interested on dynamical systems of second-order differential equations of the form

$$
\ddot{q}^{a}=\omega^{a}(t, q, \dot{q})
$$

\footnotetext{
*Email: mtsampa@phys.uoa.gr

$\dagger$ Email: anpaliat@phys.uoa.gr
} 
Eqn. (1) defines the Hamiltonian vector field $\Gamma$ in the jet space $J^{1}\left\{t, q^{a}, \dot{q}\right\}$ as follows

$$
\Gamma=\frac{d}{d t}=\frac{\partial}{\partial t}+\dot{q}^{a} \frac{\partial}{\partial q^{a}}+\omega^{a} \frac{\partial}{\partial \dot{q}^{a}} .
$$

The mathematical condition for a vector field $X^{[1]}=\xi(t, q, \dot{q}) \partial_{t}+\eta^{a}(t, q, \dot{q}) \partial_{q^{a}}+\left(\dot{\eta}^{a}-\dot{q}^{a} \dot{\xi}\right) \partial_{\dot{q}^{a}}$ in $J^{1}\left\{t, q^{a}, \dot{q}\right\}$ to be a Lie symmetry of (1) is that there exists a function $\lambda(t, q, \dot{q})$ such that

$$
\left[X^{[1]}, \Gamma\right]=\lambda(t, q, \dot{q}) \Gamma \text {. }
$$

It can be shown that the set of Lie symmetries of (1) span a Lie algebra. If the point transformation is in the base space $\left\{t, q^{a}\right\}$ that is $\xi(t, q), \eta^{a}(t, q)$ the Lie symmetry is called a point Lie symmetry. If the point transformation is in the jet space $J^{1}\left\{t, q^{a}, \dot{q}\right\}$ it is called a dynamical Lie symmetry. Usually with the term Lie symmetry one refers to the point Lie symmetries. However in the literature there have been considered many types of dynamical Lie symmetries e.g. contact symmetries, nonclassical symmetries, invariant surface symmetries and many others, (see 4, 5, 6, 7, 8, and references therein). Furthermore in dynamical Lie symmetries one has an extra degree of freedom which is removed if one demands an extra condition in which case one works with the so called gauged dynamical symmetries. In this respect one usually requires the gauge condition $\xi=0$ so that the generator is simplified to $X^{[1]}=\eta^{a}(t, q, \dot{q}) \partial_{q^{a}}+\Gamma\left(\eta^{a}\right)(t, q, \dot{q}) \partial_{\dot{q}^{a}}$. This gauge condition we shall assume in the following.

In case the dynamical equations (1) follow from a Lagrangian $L(t, q, \dot{q})$, then Emmy Noether 9 showed 1 that if a Lie symmetry of (10) leaves in addition the action integral $S=\int L(t, q, \dot{q}) d t$ invariant, that is $X(S)=\lambda S$, then there exists a function $\Phi(t, q, \dot{q})$ given by the expression

$$
\Phi(t, q, \dot{q})=\xi\left(\dot{q}^{a} \frac{\partial L}{\partial \dot{q}^{a}}-L\right)-\eta^{a} \frac{\partial L}{\partial \dot{q}^{a}}+f
$$

such that $\Gamma(\Phi)=0$, that is, $\Phi$ is a first integral of (1). Such Lie symmetries are called Noether symmetries. For a point Noether symmetry we have the additional property $X(\Phi)=\lambda \Phi$ which means that $\Phi$ is an invariant first integral. The condition for the existence of a point Noether symmetry is [10, 11]

$$
X^{[1]} L+\frac{d \xi}{d t} L=\frac{d f}{d t} .
$$

Condition (5) is called the Noether Condition. Noether point symmetries span a subalgebra of the (finite dimensional) Lie algebra of point Lie symmetries.

The function $f$ in (5) usually is refereed as "gauge" function. This is an incorrect terminology. The function $f$ is a boundary term introduced to allow for the infinitesimal changes in the value of the Action Integral produced by the infinitesimal change in the boundary of the domain caused by the transformation of the variables in the Action Integra 2. Because the dynamical equations which interest us mostly are of second order we are mainly interested for quadratic first integrals.

The main use of Noether symmetries is to provide the Noether integrals which are used to simplify the system of equations and, if there are enough of them, even to determine the solution. Necessary conditions for a Noether point symmetry are a. that there exists a Lagrangian function $L(t, q, \dot{q})$ which describes the field equations, and b. that conditions (5) are satisfied for the given Lagrangian. However, the definition of a Lagrangian for a given dynamical system is not unique. That is, it is possible that there exist non-equivalent (i.e. not differing by a perfect differential) Lagrangians describing the same dynamical equations which have different Noether symmetries (see [14, 15, 16). Therefore it is clear that when we refer to a Noether symmetry of a given dynamical system we should mention always the Lagrangian function we are assuming. More recently there have been proposed other approaches [17, 18, 19, 20, 21, 22, 23, 24] to determine first integrals of dynamical equations. Some of the authors call the resulting first integrals non-Noetherian.

For instance, the quadratic first integral of the Kepler problem, the Runge-Lenz vector, can be constructed by the first-order invariant of a scale symmetry vector [19, while Crampin in [18] calls the Runge-Lenz vector

\footnotetext{
${ }^{1}$ For an alterantive proof of the Emmy Noether's first theorem we refer the reader to [12].

${ }^{2}$ An interesting discussion on the Noether's theorem which clarifies various misinterpretations in the litterature can be found in 13.
} 
a non-Noetherian hidden symmetry. However the characterization of the Runge-Lenz vector as non-Noetherian conservation law is not accurate. The Runge-Lenz vector cannot be constructed directly from formula (4) with the use of point Noether symmetries for the classical Lagrangian of the Kepler problem. However it is derived if one uses instead contact symmetries [25]. Another classical example is the Lewis invariant of the Ermakov-Pinney system [26, 27, 28] where there are more than one approaches to reach the same result. The Runge-Lenz and the Lewis invariant differ from the invariants of the (angular) momentum in the sense that they are quadratic conservation laws.

Our approach is summarized as follows. Consider a function $I\left(t, q^{a}, \dot{q}^{a}\right)$ which is linear and quadratic in the velocities with coefficients which depend only on the coordinates $t, q^{a}$. Demand that $I$ satisfies the condition of being a first integral, that is,

$$
\frac{d I}{d t}=0
$$

Using the dynamical equations determine the resulting conditions for the coefficients of $I$ whose solution will give all possible quadratic first integrals of this form of the given dynamical equations. In case the dynamical equations follow from a regular Lagrangian define the kinetic metric $\gamma_{i j}=\frac{\partial^{2} L}{\partial \dot{q}^{2} \dot{q}}$ and consider the gauge $\xi=0$ and compute the remaining component $\eta^{i}$ of the generator of the point transformation from the Cartan condition

$$
\eta^{i}\left(t, q^{i}, \dot{q}^{i}\right)=-\gamma^{i j} \frac{\partial I_{2}}{\partial \dot{q}^{j}} .
$$

Then the symmetry vector which generates the Noether symmetry which admits this first integral is

$$
X^{[1]}=\eta^{i} \frac{\partial}{\partial q^{i}}+\boldsymbol{\Gamma}\left(\eta^{i}\right) \frac{\partial}{\partial \dot{q}^{i}}
$$

where $\Gamma$ is the Euler vector field introduced in (2). Because we can always consider the Lagrangian to be the kinetic energy, this interpretation of a first integral in terms of an associated Noether symmetry appears to be always possible. As will be shown our result generalizes those of [33, 34, for point symmetries and [18, 25, for a family of quadratic first integrals.

A further point we shall consider is the relation between the symmetries generating the quadratic integrals and the symmetries of the geometry of the kinetic metric defined by the dynamical system. This metric defines a geometry in the solution space which admits certain geometric symmetries which are called collineations. It is natural one to expect that there must be a relation between the Lie and the Noether symmetries of the dynamical equations and the collineations of the kinetic metric.

Early studies of this problem have been done by Katzin \& Levine [29, 30, Aminova [31, 32] and others. A more recent study which gives the complete answer for the case of point symmetries has been done in 33. 34 where it has been shown that the Lie point symmetries of the dynamical equations are given by the special projective algebra of the kinetic metric and the Noether point symmetries by the Homothetic algebra of the kinetic metric. Such a firm geometric result does not exist for the dynamical Lie/Noether symmetries. Similar results have been derived for second-order partial differential equations [35, 36, 37, 38. and for regular perturbative Lagrangians [39]. Furthermore results for singular Lagrangians which support constrained dynamical systems can be found in 40, 41.

The plan of the paper is as follows. In Section 2 are given the basic properties of collineations and Killing tensors of Riemannian manifolds. Some new results are given which enable one to construct Killing tensors of order 2 from the collineations. In section 3 we discuss the relation between collineations of the kinetic metric and quadratic first integrals. Finally, in Section 4 we discuss our results and draw our conclusions.

\section{Collineations and Killing tensors of second-order}

Consider a Riemannian space $V^{n}$ with metric tensor $g_{a b}$. Let $\boldsymbol{\Omega}\left(x^{c}\right)$ be a linear geometric object $3^{3}$ in $V^{n}$ which under the action of the infinitesimal transformation $x^{a^{\prime}}=x^{a^{\prime}}\left(x^{b}, \varepsilon\right)$ transforms as follows

$$
\overline{\boldsymbol{\Omega}}\left(\boldsymbol{\Omega}, x^{c}, x^{c^{\prime}}\right)=J\left(x^{c}, x^{c^{\prime}}\right) \ldots J\left(x^{c}, x^{c^{\prime}}\right) \boldsymbol{\Omega}+\boldsymbol{\Xi}\left(x^{c}, x^{c^{\prime}}\right),
$$

\footnotetext{
${ }^{3}$ With the term "geometric object" we mean a quantity in $V^{n}$ associated with a transformation rule. Not necessarily a tensor field.
} 
where $J\left(x^{c}, x^{c^{\prime}}\right)$ denotes the Jacobian matrix of the transformation.

A collineation of the geometric object $\Omega\left(x^{c}\right)$ under the action of the infinitesimal transformation $x^{a^{\prime}}=$ $x^{a^{\prime}}\left(x^{b}, \varepsilon\right)$ generated by the vector field $X\left(x^{c}\right)$ is defined by

$$
\boldsymbol{\Psi}\left(x^{c}\right)=\lim _{\varepsilon \rightarrow 0} \frac{\overline{\boldsymbol{\Omega}}\left(\boldsymbol{\Omega}, x^{a^{\prime}}\left(x^{b}, \varepsilon\right)\right)-\boldsymbol{\Omega}\left(x^{b}\right)}{\varepsilon} .
$$

where $\Psi$ has the same numbers of components and symmetries of the indices with $\Omega$.The rhs of the latter expression is the definition of the Lie derivative wrt $X$ therefore (10) is written in the simple form $\mathcal{L}_{X} \boldsymbol{\Omega}=\boldsymbol{\Psi}$.

Collineations involving geometric objects derived from the metric tensor, $g_{a b}$, i.e. the Christofell symbols $\Gamma_{b c}^{a}$, the Ricci tensor etc. play an important role in the mathematical structure of the Riemannian manifold $V^{n}$ and on the properties of the physical systems defined on $V^{n}$.

\subsection{Collineations concerning the metric tensor}

In the present work of special interest are two families of geometric collineations. The first family are the conformal motions defined by the requirement

$$
\mathcal{L}_{X} g_{a b}=2 \psi\left(x^{c}\right) g_{a b}
$$

in which the vector field $X$ is called a Conformal Killing Vector (CKV) and the function $\psi\left(x^{c}\right)$ the conformal factor. One shows easily that $\psi=\frac{1}{\operatorname{dim} V^{n}} X^{c}{ }_{; c}$. In case $\psi_{; i j}=0, \psi_{, i}=0$ with $\psi \neq 0$ and $\psi=0$ the CKV specializes to a special CKV, a homothetic vector (HV) and to a Killing vector (KV) respectively.

The second family are the projective collineations (PC) defined by the requirement

$$
\left.L_{X} \Gamma_{b c}^{a}=2 \delta_{(b}^{a} \phi, c\right)
$$

where $\phi\left(x^{c}\right)$ is a general function. In case $\phi_{; i j}=0$ with $\phi_{, i} \neq 0$ and $\phi=0$ the PC specializes to a special PC and an Affine collineation (AC) respectively. An AC which is not a KV or the $\mathrm{HV}$ is called a proper AC.

Using the identity $L_{\eta} \Gamma_{b c}^{a}=\eta_{; b c}^{a}-R_{b c d}^{a} \eta^{d}$, one rewrites condition (12) as follows

$$
\left.X_{;(b c)}^{a}-2 \delta_{(b}^{a} \phi, c\right)=R_{b c d}^{a} X^{d}
$$

from which follows that

$$
\left.X_{(a ; b}-2 g_{a b} \phi\right)_{; c)}=0 .
$$

A Killing tensor (KT) $K$ is defined by the requirement

$$
\left[K, g_{a b}\right]_{S N}=0
$$

or equivalently

$$
K_{\left(a_{1} \ldots a_{k} ; c\right)}=0
$$

where $[,]_{S N}$ denotes the Schouten-Nijenhuis bracket, a generalization of the Lie derivative. If $K$ is a vector field then $[,]_{S N}$ reduces to the Lie derivative and the KT reduces to a KV. From (14) it follows that the second-rank tensor $C_{a b}=X_{(a ; b)}-2 g_{a b} \phi$ is a Killing tensor of rank two. Therefore it is clear that PCs can be used to construct KTs.

In the special case that the $\mathrm{KT} C_{a b}$ comes form a vector field $L^{a}$ such that $C_{a b}=L_{(a ; b)}$, we can define the vector field $M_{a}=L_{a}-X_{a}$, where $X^{a}$ is a PC which satisfies the relation

$$
M_{(a ; b)}=-2 \phi g_{a b},
$$

that is, $M^{a}$ is a CKV with conformal factor $-\phi$.

Therefore if in a space there are $m \mathrm{CKVs} M^{a}$ and $m$ PCs $X^{a}$ such that the conformal factor is minus the projection factor, we construct $m$ KTs of order two of the form $C_{a b}=L_{(a ; b)}$ where $L_{a}=M_{a}+\eta_{a}$.

From condition (14) it follows that an $\mathrm{AC} \eta^{a}$ satisfies

$$
\eta_{(a ; b c)}=0
$$

therefore $C_{a b}=\eta_{(a ; b)}$ defines the KT of order two. We conclude that from the $s$ (say) proper ACs $n^{a}$ of a space we construct the $s$ KTs $C_{a b}=\eta_{(a ; b)}$. 


\section{$2.2 \quad$ Killing tensors and collineations}

Concerning the KTs on a finite dimensional Riemannian space $V^{n}$ we have the following results. Let $V^{n}$ be a Riemannian or Pseudo-Riemannian manifold of dimension $n$. Then:

1. The KTs of rank $m$ defined on $V^{n}$ form a linear space of dimension less than or equal to

$$
\frac{(n+m-1) !(n+m) !}{(n-1) ! m ! n !(m+1) !}
$$

and the equality is attained if and only if $V^{n}$ is a space of constant curvature [42.

2. The maximum number of linearly independent Killing Tensors of order 2 in a Riemannian manifold of dimension $n$ is $n(n+1)^{2}(n+2) / 12$ and this is the necessary and sufficient condition for the space to be of constant curvature 43, 44, 45.

3. On flat spaces the Killing tensors are all reducible which means that all are generated by products of Killing vectors [46].

From the above results we conclude the following concerning the KTs of order 2 defined on $V^{n}$.

a. If the space admits $m$ gradient $\mathrm{KVs} S_{J}(J=1,2, \ldots, m)$ and $r$ non-gradient $\mathrm{KVs} M_{A}(A=1,2, \ldots, r)$ then we can construct $m^{2}+m r=m(m+r)$ KTs of rank two (excluding the metric), of the form $C_{a b}=L_{(a ; b)}$ where

$$
L_{a}=S_{I} S_{J, a}+S_{I} M_{A a} .
$$

b. If the space admits another $k$ proper PCs $n^{a}$ with projection factor $\phi$ and $k$ CKVs $G_{a}$ with conformal factor $-\phi$ then we may construct another $k$ KTs of rank two of the same form $C_{a b}=L_{(a ; b)}$ where

$$
L_{a}=G_{a}+\eta_{a} .
$$

Finally if the space admits $r$ proper ACs then we may construct another $r$ KTs.

We collect these results in the following

Proposition 1 In a space $V^{n}$ the vector fields of the form

$$
L_{a}=c_{1} K V_{a}+c_{2} P C_{a}+c_{3} A C_{a}+c_{1 I} S_{I, a}+c_{2 I J} S_{I} S_{J, a}+2 c_{I A} S_{I} M_{A a}
$$

where the proper PCs are CKVs such that the conformal factor is minus the projective factor, the $A C_{a}$ are proper and $K V_{a}$ are the non-gradient $K V$ s produce $K T s$ of order 2 of the form $C_{a b}=L_{(a ; b)}$. In the case of maximally symmetric spaces there do not exist proper PCs and proper ACs therefore only the vectors generated by the KVs are necessary, that is, in these spaces

$$
L_{a}=c_{1} K V_{a}+c_{1 I} S_{I, a}+c_{2 I J} S_{I} S_{J, a}+2 c_{3 I A} S_{I} M_{A a} .
$$

The KVs give the solution $C_{a b}=0$ and the $H V$ gives the solution $C_{a b}=g_{a b}$. These vectors construct all KTs $C_{a b}$ of a flat space. The vectors $S_{I} S_{J, a}$ and $S_{I} M_{A a}$ are gradient and non-gradient non-proper ACs respectively.

It is important to note that in general not all KTs of order 2 in (a non-flat) $X^{n}$ are reducible, that is of the form $C_{a b}=L_{(a ; b)}$.

Let us apply the above proposition to the case of $E^{2}$ and reconstruct the KTs. The $E^{2}$ admits two gradient $\mathrm{KVs} \partial_{x}, \partial_{y}$ whose generating functions are $x, y$ respectively and one non-gradient $\mathrm{KV}$ (the rotation) $y \partial_{x}-x \partial_{y}$. From the KVs we can construct $2(2+1)=6$ proper KTs. From (19) we have

$$
L_{a}=c_{11}\left(\begin{array}{l}
1 \\
0
\end{array}\right)+c_{12}\left(\begin{array}{l}
0 \\
1
\end{array}\right)+c_{211} x\left(\begin{array}{l}
1 \\
0
\end{array}\right)+c_{212} x\left(\begin{array}{l}
0 \\
1
\end{array}\right)+c_{221} y\left(\begin{array}{l}
1 \\
0
\end{array}\right)+c_{222} y\left(\begin{array}{l}
0 \\
1
\end{array}\right)+2 c_{311} x\left(\begin{array}{c}
y \\
-x
\end{array}\right)+2 c_{312}\left(\begin{array}{c}
y \\
-x
\end{array}\right)
$$


or in a more convenient form

$$
L_{a}=c_{11}\left(\begin{array}{l}
1 \\
0
\end{array}\right)+c_{9}\left(\begin{array}{l}
0 \\
1
\end{array}\right)+A x\left(\begin{array}{l}
1 \\
0
\end{array}\right)+B y\left(\begin{array}{l}
0 \\
1
\end{array}\right)+c_{8} y\left(\begin{array}{l}
1 \\
0
\end{array}\right)+c_{10} x\left(\begin{array}{l}
0 \\
1
\end{array}\right)+2 a x\left(\begin{array}{c}
y \\
-x
\end{array}\right)-2 \beta y\left(\begin{array}{c}
y \\
-x
\end{array}\right)
$$

which can be written

$$
L^{a}=\left(\begin{array}{c}
A x+2 a x y+2 \beta x^{2}+c_{8} y+c_{11} \\
B y+2 a y^{2}+2 \beta x y+c_{10} x+c_{9}
\end{array}\right) .
$$

The generic form of the tensor $C_{a b}=L_{(a ; b)}$ in $E^{2}$ is

$$
C_{a b}=\left(\begin{array}{ll}
L_{x x} & \frac{1}{2}\left(L_{x y}+L_{y x}\right) \\
\frac{1}{2}\left(L_{x y}+L_{y x}\right) & L_{y y}
\end{array}\right)=\left(\begin{array}{ll}
A+2 a y & C-a x-\beta y \\
C-a x-\beta y & B+2 \beta y
\end{array}\right)
$$

where for the constant $C$ we have the constraint $C=\frac{c_{8}+c_{10}}{2}$.

\section{Quadratic conservation laws and collineations}

Consider a dynamical system defined by the second-order differential equation

$$
\ddot{q}^{a}+\Gamma_{b c}^{a} \dot{q}^{b} \dot{q}^{c}+Q^{a}\left(t, q^{c}\right)=0 .
$$

where $\Gamma_{b c}^{a}\left(q^{c}\right)$ are the Christoffel symbols defined by the kinetic metric (i.e. the kinetic energy) of the system. The external forces $Q^{a}$ are given by

$$
Q_{a}=V_{, a}-F_{a}
$$

where $V^{a}$ stands for the total potential $V\left(t, q^{c}\right)$ defined by the conservative forces and $F^{a}$ stands for the sum of the non-conservative forces. For this system we may consider the Lagrange function (assumed to be regular )

$$
L\left(t, q^{c}, \dot{q}^{c}\right)=\frac{1}{2} g_{a b}\left(q^{c}\right) \dot{q}^{a} \dot{q}^{b}-V\left(t, q^{c}\right) .
$$

and write (23) in the form $E_{a}(L)=Q_{a}$ where $E_{a}=\frac{d}{d t} \frac{\partial}{\partial \dot{q}^{a}}-\frac{\partial}{\partial q^{a}}$ is the Euler-Lagrange vector.

We consider next the function

$$
I=K_{a b}\left(t, q^{c}\right) \dot{q}^{a} \dot{q}^{b}+K_{a}\left(t, q^{c}\right) \dot{q}^{a}+K\left(t, q^{c}\right),
$$

which is the quadratic in the velocities and demand that it is a first integral, that is, satisfies the equation $\frac{d I}{d t}=0$ modulo the equations of motion (23). This approach provides us the first integrals of this form. This approach relies only on the dynamical equations (23) and not on the Noether theorem which requires the (non-unique) Lagrangian function. This idea is not new. It appears that is was raised for the first time by Darboux [47] and later by Wittaker 48 , who both considered the case of Newtonian autonomous holonomic systems with two degrees of freedom and determined most potentials $V(q)$ for which these systems admit a quadratic first integral other than the Hamiltonian (energy). The complete answer to this problem was given much later by G. Thompson [49, for some extensions see also [50, 51, 52] and references therein.

The requirement $\frac{d I}{d t}=0$ leads to the equation

$$
\begin{aligned}
0 & =K_{(a b ; c)} \dot{q}^{a} \dot{q}^{b} \dot{q}^{c}+\left(K_{a b, t}+K_{a ; b}\right) \dot{q}^{a} \dot{q}^{b} \\
& +\left(K_{a, t}+K_{, a}\right) \dot{q}^{a}-2 K_{a r} \delta_{b}^{(r} F^{a)} \dot{q}^{b} \\
& +K_{, t}-K_{a} F^{a}
\end{aligned}
$$

from which using the various powers of the velocity follow the conditions

$$
\begin{gathered}
K_{(a b ; c)}=0 \\
K_{(a ; b)}=-K_{a b, t}
\end{gathered}
$$




$$
\begin{gathered}
K_{, b}+K_{b, t}-2 K_{a b} F^{a}=0 \\
K_{, t}=K_{a} F^{a} .
\end{gathered}
$$

It is important to mention here, that these conditions reduce to the Noether condition (5) when $F^{a}=0$ and $X=\left(K_{b}^{a} \dot{q}^{b}+K^{a}\right) \partial_{a}$ and to the weak Noether condition

$$
X^{[1]} L+\frac{d \xi}{d t} L+\eta^{a} Q_{a}=\frac{d f}{d t} .
$$

which also known as Noether Bessel Hagen equation (NBH) [53] when $F^{a} \neq 0$.

The "solution "of conditions (28) - (31) will provide all quadratic first integrals of (23) for the form (26).

Condition (28) implies that $K_{a b}$ is a KT for the kinetic metric $\gamma_{a b}$. These conditions have been considered previously and they have been "solved " for special cases. For example Kalotas et al.[25] considered the case for $F^{a}=0$ and $K^{a}=0$.

On the other hand in [54] they have considered the case $K^{a} \neq 0$, and $K_{a b}=g(t) \gamma_{a b}$ for point symmetries, where $K^{a}$ is a HV or a KV of the metric $\gamma_{a b}$ and $g_{, t}$ is the homothetic factor of $K^{a}$.

In the general scenario considered here, $K_{a b}$ is a $\mathrm{KT}$ of the form $K_{a b}(t, q)=g(t) C_{a b}(q)$ where $C_{a b}\left(q^{a}\right)$ is a general KT of the kinetic metric. Then from (29) it follows that

$$
K_{a}(t, q)=f(t) L_{a}(q)+B_{a}(q),
$$

from where the symmetry conditions become

$$
\begin{gathered}
f(t) L_{(a ; b)}+B_{(a ; b)}+g_{, t} C_{a b}=0, \\
K_{, b}+f_{, t} L_{b}-2 g(t) C_{a b} F^{a}=0, \\
f(t) L_{a} F^{a}+B_{a} F^{a}-K_{, t}=0 .
\end{gathered}
$$

From the discussion of the previous section we can see that equation (34) is again a geometric condition. It relates the $\mathrm{KT} C_{a b}$ with the vector fields $L^{a}$ and $B^{b}$. The latter vector fields are given from formulas (18) or (19) of Proposition 1.

We conclude that the quadratic conservation laws (26) with nonvanishing linear term, exists if and only if the Riemannian space admits a nontrivial projective algebra, which means that at least, a reducible KT exists given by the geometric collineations as discussed in Section 2

\subsubsection{Application}

In order to demonstrate the application of our geometric results let us consider a dynamical system consists by the following differential equations

$$
\ddot{x}=F_{1}(x, y) \text { and } \ddot{y}=F_{2}(x, y)
$$

where $F_{1}(x, y), F_{2}(x, y)$ are arbitrary functions. For the later system we observe that the underlying geometry is that of the two-dimensional Euclidean space. Consider now, that conditions (34)-(36) are satisfied where $B^{a}=y \partial_{x}-x \partial_{y}$ and $L^{a}=x \partial_{x}$. Hence from (34) it follows that $C_{a b}=\left(\begin{array}{cc}c_{0} & 0 \\ 0 & 0\end{array}\right)$ with $g_{, t}=\frac{1}{c_{0}} f$.

Therefore, conditions (35), (36) provides

$$
\begin{gathered}
K_{, x}+c_{0} g_{, t t} x-2 g(t) F_{1}(x, y)=0, \quad K_{, y}=0, \\
c_{0} g_{, t}\left(F_{1}(x, y)\right)+y F_{1}(x, y)-x F_{2}(x, y)-K_{, t}=0 .
\end{gathered}
$$

We assume that $g_{, t}=0$, that is $g(t)=\frac{1}{c_{0}}$, then the latter system is simplified as

$$
\begin{gathered}
K_{, x}-\frac{2}{c_{0}} F_{1}(x, y)=0, \quad K_{, y}=0, \\
\left(F_{1}(x, y)\right)+y F_{1}(x, y)-x F_{2}(x, y)-K_{, t}=0 .
\end{gathered}
$$


which gives that $K=v(x)+n(t)$, and

$$
F_{1}(x, y)=\frac{1}{2 c_{0}} v_{, x}(x) \text { and } F_{2}(x, y)=c_{0} \frac{y v(x)_{, x}}{2 x}+\frac{n_{, t}(t)}{x} .
$$

The latter means that all the dynamical systems of the form

$$
\ddot{x}=\frac{c_{0}}{2} v_{, x}(x), \ddot{y}=\frac{c_{0} y v(x)_{, x}+2 n_{, t}(t)}{2 x}
$$

admit the quadratic conservation law

$$
I=-\frac{1}{c_{0}} \dot{x}^{2}+y \dot{x}-x \dot{y}+v(x)+n(t) .
$$

Working in the same way one is possible to classify all the types of dynamical systems of the generic form (37) which admit quadratic conservation laws and that without making use of Noether's theorem or other type of symmetries but the collineations of the kinetic metric. Essentially the solution of the equation $d I / d t=0$ is a matter of geometry.

\section{Conclusions}

The relation between the collineations of the kinetic metric and the existence of first integrals for autonomous quasilinear differential equations has been discussed. We reviewed previous results in the literature and derived a system of equations whose solution derives all the quadratic first integrals they admit.

The purpose of our discussion was to make clear that first integrals for a given dynamical system do not follow necessary from Noether's theorem but they are rather a matter of geometry. Moreover, the characterization of some first integrals as non-Noetherian is also meaningless.

In particular for a given dynamical system a geometry can be defined via the kinetic metric and the existence of geometric collineations of the kinetic metric is the only necessary and sufficient condition for first integrals to exist. In a sense a dynamical system is somehow "constrained" by the geometry it creates, because the collineations of this geometry provide the first integrals for the dynamical and consequently specify its evolution.

The new mathematical relations which discussed in Section 2 on the construction of KTs with the use of the PCs indicate the importance of the geometry for the existence of Lie/Noether symmetries. This means, that working with collineations one is possible to extract information for the existence of quadratic first integrals without performing the standard formal calculations required by the Lie approach.

In a forthcoming work we plan to extend the geometric analysis on the symmetry conditions (34) - (36) in order to understand in detail how geometry is related to the force-term $F^{a}$. Such an analysis will give us impor-

tant information for the geometric properties of integrable models and also a possible geometric classification for the separable and non-separable Hamiltonian systems, for instance see [55. Equally important step is the general solution of the system of equations (34) - (36) which will provide all quadratic first integrals of (23) of the form (26).

\section{Acknowledgements}

The research of AP was supported by FONDECYT postdoctoral grant no. 3160121. AP thanks the University of Athens for the hospitality provided this work was carried out.

\section{References}

[1] S. Lie, Theorie der Transformationsgruppen I, Leipzig: B. G. Teubner (1888)

[2] S. Lie, Theorie der Transformationsgruppen II, Leipzig: B. G. Teubner (1888)

[3] S. Lie, Theorie der Transformationsgruppen III, Leipzig: B. G. Teubner (1888)

[4] G. Bluman, Math. Com. Mod. 18, 1 (1993) 
[5] V. Grassi, R.A. Leo, G. Soliani and T. Tempesta, Physica A: Stat. Mech. Appl. 286, 79 (2000)

[6] P.J. Olver, J. Non. Math. Phys. 9, 164 (2002)

[7] P.A. Clarkson, Math. Com. Mod. 18, 45 (1993)

[8] K.S. Govinder and P.G.L. Leach, J. Phys. A: Math. Gen. 28, 5349 (1995)

[9] E. Noether, Nachr. d. König. Gesellsch. d. Wiss. zu Göttingen, Math-phys. Klasse, 235, (1918) (translated in English by M.A. Tavel physics/0503066])

[10] W. Sarlet and F. Cantrijin, J. Phys. A: Math. Gen. 14, 479 (1981)

[11] W. Sarlet and F. Cantrijin, SIAM Review 23, 467 (1981)

[12] G.H. Katzin and J. Levine, J. Math. Phys. 17, 1345 (1976)

[13] G.P. Flessas, P.G.L. Leach and S. Cotsakis, Can. J. Phys. 73, 543 (1995)

[14] M.C. Nucci and P.G.L. Leach, J. Non. Math. Phys. 9, 110 (2002)

[15] M.C. Nucci and P.G.L. Leach, Phys. Scr. 78, 065011 (2008)

[16] M.C. Nucci and K.M. Tamazhmani, J. Non. Math. Phys. 17, 167 (2010)

[17] S.A. Hojman, J. Math. Phys. A: Math. Gen. 24, L291 (1992)

[18] M. Crampin, Rep. Math. Phys. 20, 31 (1984)

[19] G. Prince, C. Eliezer, J. Phys. A: Math. Gen. 13, 815 (1980)

[20] P.G.L. Leach and V.M. Govinder, Lett. A 133, 289 (1988)

[21] C. Clementi and M. Pettini, Celest. Mech. Dyn. Astron. 84, 263 (2002)

[22] B.D. Vujanovic, Int. J. Non-Linear Mech. 30, 783 (1995)

[23] B. Vujanovic, Int. J. Non-Linear Mech. 13, 185 (1978)

[24] S. Moyo and P.G.L. Leach, J. Phys. A: Math. Gen. 35, 5333 (2002)

[25] T.M. Kalotas and B.G. Wybourne, J. Phys. A: Math. Gen. 15, 2077 (1982)

[26] H.R. Lewis, Phys. Rev. Lett. 18, 510 (1967)

[27] M. Tsamparlis and A. Paliathanasis, J. Phys. A: Math. Theor. 45, 275202 (2012)

[28] S. Moyo and P.G.L. Leach, J. Phys. A: Math. Gen. 35, 5333 (2002)

[29] G.H. Katzin and J. Levine, J. Math. Phys. 9, 8 (1968)

[30] G.H. Katzin and J. Levine, J. Math. Phys. 22, 1878 (1981)

[31] A.V. Aminova, Sbornik Math. 186, 1711 (1995)

[32] A.V. Aminova, Tensor N.S., 65, 62 (2000)

[33] M. Tsamparlis and A. Paliathanasis, Nonlinear Dynamics 62, 203 (2010)

[34] M. Tsamparlis and A. Paliathanasis, Gen. Relativ. Gravit. 42, 2957 (2010)

[35] A. Paliathanasis and M. Tsamparlis, J. Geom. Phys. 62, 2443 (2012)

[36] A. Paliathanasis and M. Tsamparlis, J. Geom. Phys. 107, 45 (2016)

[37] A. Paliathanasis and M. Tsamparlis, J. Geom. Phys. 124, 165 (2018) 
[38] Y. Bozhkov and I.L. Freire, J. Diff. Eq. 249, 872 (2010)

[39] A. Paliathanasis and S. Jamal, J. Geom. Phys. 124, 300 (2018)

[40] T. Christodoulakis, N. Dimakis and P.A Terzis, J. Phys. A 47, 095202 (2014)

[41] P.A. Terzis, N. Dimakis, T. Christodoulakis, A. Paliathanasis and M. Tsamparlis, J. Geom. Phys. 101, 52 (2016)

[42] E. G. Kalnins and W. M. Miller, SIAM J. Math. Anal. 11, 1001 (1980)

[43] T.Y. Thomas, Proc N.A.S. 32, 10 (1946)

[44] C.D.J. Collinson, J. Phys. A: Gen. Phys. 4, 756 (1965)

[45] D. Garfinkle and E.N. Glass, Class. Quantum Grav. 27, 095004 (2010)

[46] G. Thompson, J. Math. Phys. 27, 2693 (1986)

[47] G. Darboux, Archives Neerlandaises 6, 371 (1901)

[48] E.T. Whittaker, A Treatise on the Analytical Dynamics of Particles and Rigid Bodies (Cambridge Mathematical Library) (1937)

[49] G. Thompson, J. Phys. A: Math. Gen. 17, 985 (1984)

[50] C. Daskaloyannis and Y. Tanoudis, J. Math. Phys. 48, 072108 (2007)

[51] E.G. Kalnins, J.M. Kress, W. Jr. Miller and S. Post, Sigma 5, 008 (2009)

[52] Y. Chen, E.G. Kalnins and W. Jr. Miller, Sigma 11, 088 (2015)

[53] D. Djukic, Int. J. Non-linear Mechanics 479, (1973)

[54] L. Karpathopoulos, A. Paliathanasis and M. Tsamparlis, J. Math. Phys. 58, 082901 (2017)

[55] E.G. Kalnins, Seperation of variables for Riemannian spaces of constant curvature, Longman Scientific \& Technical, New York, (1986) 